\title{
ASYMPTOTIC STABILITY, INSTABILITY AND STABILIZATION OF RELATIVE EQUILIBRIA
}

\author{
A. M. Bloch ${ }^{1}$ \\ Department of Mathematics \\ The Ohio State University \\ Columbus, $\mathrm{OH} 43210$
}

\author{
J. E. Marsden ${ }^{3}$ \\ Dept. of Mathematics \\ University of California \\ Berkeley, CA 94720
}

\author{
P. S. Krishnaprasad ${ }^{2}$ \\ Dept. of Electrical Engineering and \\ Systems Research Center \\ University of Maryland \\ College Park, MD 20742
}

\author{
T. S. Ratiu ${ }^{4}$ \\ Dept. of Mathematics \\ University of California \\ Santa Cruz, CA 95064
}

\section{Abstract}

In this paper we analyze asymptotic stability, instability and stabilization for the relative equilibria, i.e. equilibria modulo a group action, of natural mechanical systems. The practical applications of these results are to rotating mechanical systems where the group is the rotation group. We use a modification of the Energy-Casimir and Energy-Momentum methods for Hamiltonian systems to analyze systems with dissipation. Our work couples the modern theory of block diagonalization to the classical work of Chetaev.

\section{INTRODUCTION}

A central problem in control theory is that of stabilizing nonlinear systems. Very often the systems one is interested in are mechanical systems, which, in the absence of dissipation, are Hamiltonian in nature. Further, the equilibria we wish to stabilize are relative equilibria-equilibria modulo a group action, usually the rotation group. Recently two distinct but related methods have been developed to analyze the stability of the relative equilibria of Hamiltonian systems. The first, the "EnergyCasimir" method, originally goes back to Arnold [2] and was developed and formalized in Holm, Marsden, Ratiu and Weinstein [8] and Krishnaprasad

\footnotetext{
${ }^{1}$ Supported in part by NSF Grant DMS-90-02136 and a Seed Grant from Ohio State University.

2 Supported in part by AFOSR-87-0073 and AFOSR-900105 and NSF Engineering Resarch Program grant AFOSR87-0073.

3 Supported in part by DOE Contract DE-FG03-88ER25064 .

${ }^{4}$ Supported in part by NSF Grant DMS-8922699.
}

and Marsden [10] and related papers. While the analysis in this method takes place in the body frame, the analysis in the "Energy-Momentum" method takes place in the material frame - see Marsden, Simo, Lewis and Posbergh [11] and Simo, Posbergh and Marsden [14]. Both these techniques use a combination of energy and other conserved quantitites to prove nonlinear (Lyapunov) stability.

In the papers Bloch and Marsden [4] and Bloch, Krishnaprasad, Marsden and Sanchez de Alvarez [5] we showed that for some systems the Hamiltonian structure could be preserved under feedback enabling these techniques to be used to analyze feedback stabilization.

In this paper we consider asymptotic stability and instability results associated with the EnergyCasimir and Energy-Momentum methods. We consider first the problem of stabilizing a spacecraft (rigid body) with momentum wheels. We show that when the Energy-Casimir method gives nonlinear stability, the addition of velocity feedback yields asymptotic stability, as demonstrated by a RouthHurwitz analysis. (See also [15].) This indicates how one can use Hamiltonian methods in asymptotic stabilization - first one achieves nonlinear stability in the Hamiltonian context then one adds a dissipative velocity feedback.

We then turn to the question of instability. Our main point of interest here is to determine if a nonlinear system becomes unstable if the Lyapunov function in the Energy-Casimir or Energy-Momentum methods becomes indefinite. We do the analysis here in the context of the Energy-Momentum method as it has been shown - see Marsden et. al. [11], Simo et. al. [14] - that this method yields a normal form for the linearized equations of motion that may be analyzed in quite general fashion. 
This normal form corresponds to a block diagonalization of the second variation of the energymomentum function. For the case of $S^{1}$ symmetry it turns out that the normal form is precisely that of the general linear mechanical system with gyroscopic forces analyzed by Chetaev [6]. We discuss Chetaev's result that a gyroscopically stabilized system (i.e. a system that is unstable in the absence of gyroscopic forces) becomes unstable in the presence of damping. In the two degree of freedom case we show how this can be demonstrated by a Routh analysis. In the general case this can be done by Chetaev's Lyapunovtype analysis. We also discuss extensions to infinite dimensions and the application of a theorem due in different contexts to Chetaev [6], Oh [12] and others - relating the oddness of the number of eigenvalues of the Hessian of the Lyapunov function to spectral instability.

\section{The Energy-Casimir Method and Asymptotic Stabilization of a Dual Spin Sattelite.}

In this section we describe briefly the EnergyCasimir method for stabilization. We then describe how it was used (see Bloch et. al [5]) to show how a dual spin satellite can be stabilized about its intermediate axis of inertia by quadratic feedback in a single rotor. We demonstrate by the Routh scheme that the addition of velocity feedback to the rotor then gives asymptotic stability. (An alternate approach is discussed in [9].)

The Energy-Casimir method is as follows: Write the equations of motion (in the body frame) as $\dot{u}=$ $F(u)$ on a given space $P$. Find a conserved function $H$ for the system. $H$ is usually taken to be the Hamiltonian and the equations are in Hamiltonian form $\dot{F}=\{F, H\}$ where $\{$,$\} is the Lie-Poisson$ bracket on $P$, a Poisson manifold. Then find a family of constants of motion $C$ for the system. Often these are taken to be Casimirs - functions that commute with every other function under the bracket. Now choose $C$ such that $H+C$ has a critical point at the equilibrium $u_{e}$ of interest. Finally show that the second variation of $H+C$ is definite at $u_{e}$. This proves Lyapunov stability (in finite dimensions - in infinite dimensions some a priori estimates are needed).

Consider now the equations for a rigid body with a single rotor. Let the rigid body have moments of inertia $I_{1}>I_{2}>I_{3}$ and suppose the symmetric rotor is aligned with the third principal axis and has moments of inertia $J_{1}=J_{2}$ and $J_{3}$. Let $\omega_{i}, i=1,2,3$, denote the carrier body angular velocities and let $\dot{\alpha}$ denote that of the rotor (relative to a frame fixed on the carrier body). Let

$$
\begin{aligned}
& \operatorname{diag}\left(\lambda_{1}, \lambda_{2}, \lambda_{3}\right) \\
& =\operatorname{diag}\left(J_{1}+I_{1}, J_{2}+I_{2}, J_{3}+I_{3}\right)
\end{aligned}
$$

be the locked inertia tensor. The natural momenta for the system are:

$$
\begin{aligned}
m_{i} & =\left(J_{i}+I_{i}\right) \omega_{i}=\lambda_{i} \quad i=1,2 \\
m_{3} & =I_{3} \omega_{3}+\ell_{3} \\
\ell_{3} & =J_{3}\left(\omega_{3}+\dot{\alpha}\right) .
\end{aligned}
$$

Then one can show (see Bloch et. al. [5]) that the equations of motion are

$$
\begin{aligned}
& \dot{m}_{i}=m_{2} m_{3}\left(\frac{1}{I_{3}}-\frac{1}{\lambda_{2}}\right)-\frac{\ell_{3} m_{2}}{I_{3}} \\
& \dot{m_{2}}=m_{1} m_{3}\left(\frac{1}{\lambda_{1}}-\frac{1}{I_{3}}\right)+\frac{\ell_{3} m_{1}}{I_{3}} \\
& \dot{m_{3}}=m_{1} m_{2}\left(\frac{1}{\lambda_{2}}-\frac{1}{\lambda_{1}}\right) \\
& \dot{\ell_{3}}=u
\end{aligned}
$$

where $u$ is the rotor torque. Choosing $u=k a_{3} m_{1} m_{2}$ where $a_{3}=\left(\frac{1}{\lambda_{2}}-\frac{1}{\lambda_{1}}\right)$, the equations reduce to the system

$$
\begin{aligned}
& \dot{m_{1}}=m_{2}\left(\frac{(1-k) m_{3}-p}{I_{3}}\right)-\frac{m_{3} m_{2}}{\lambda_{2}} \\
& \dot{m_{2}}=-m_{1}\left(\frac{(1-k) m_{3}-p}{I_{3}}\right)+\frac{m_{3} m_{1}}{\lambda_{1}} \\
& \dot{m_{3}}=a_{3} m_{1} m_{2}
\end{aligned}
$$

which are Hamiltonian on $s o(3)^{*}$ with resepct to the standard Lie-Poisson structure with Hamiltonian

$$
H=\frac{1}{2}\left(\frac{m_{1}^{2}}{\lambda_{1}}+\frac{m_{2}^{2}}{\lambda_{2}}+\frac{\left((1-k) m_{3}-p\right)^{2}}{(1-k) I_{3}}\right)
$$

where $p$ is a constant integral of the motion.

Now using the Energy-Casimir function $H+C$ where $C=\varphi\left(m_{1}^{2}+m_{2}^{2}+m_{3}^{2}\right), \varphi$ a smooth function, we can prove

Theorem 2.1. For $k>1-\frac{I_{3}}{\lambda_{2}}$ (and $p=0$ ) the system (2.4) is stabilized about the relative equlibrium $(0, M, 0)$.

For details of the proof see Bloch et. al. [5].

This yields Lyapunov stability for the given equilibrium. We would like to obtain asymptotic stability. 
Let us add therefore velocity feedback $\dot{\alpha}$ in the rotor. Since $\dot{\alpha}=\frac{\ell_{3}}{J_{3}}-\omega_{3}$ this yields the equations

$$
\begin{aligned}
\dot{m_{1}}= & m_{2} m_{3}\left(\frac{1}{I_{3}}-\frac{1}{\lambda_{2}}\right)-\frac{\ell_{3} m_{2}}{I_{3}} \\
\dot{m_{2}}= & m_{1} m_{3}\left(\frac{1}{\lambda_{1}}-\frac{1}{I_{3}}\right)+\frac{\ell_{3} m_{1}}{I_{3}} \\
\dot{m_{3}}= & m_{1} m_{2}\left(\frac{1}{\lambda_{2}}-\frac{1}{\lambda_{1}}\right) \\
\dot{\ell_{3}}= & k a_{3} m_{1} m_{2} \\
& -r\left\{\ell_{3}\left(\frac{1}{J_{3}}+\frac{1}{I_{3}}\right)-\frac{m_{3}}{I_{3}}\right\}
\end{aligned}
$$

for some $k, r>0$.

To analyze stability for the system we linearize (2.4) about the given equilibrium $(0, M, 0)$. This yields a system with characteristic polynomial

$$
\begin{gathered}
\lambda\left\{\lambda^{3}+\lambda^{2} r \nu+\lambda\left[\frac{M^{2}}{I_{3}} k a_{3}-M^{2} a_{3} \mu\right]\right. \\
\left.+M^{2} a_{3}\left[-\mu r \nu+\frac{r}{I_{3}^{2}}\right]\right\}
\end{gathered}
$$

where $\mu=\left(\frac{1}{I_{3}}-\frac{1}{\lambda_{2}}\right), \nu=\left(\frac{1}{J_{3}}+\frac{1}{I_{3}}\right)$. Now for $r=$ 0 we see the system has two zero eigenvalues and eigenvalues in the left and right half-planes for $k<$ $1-\frac{I_{3}}{\lambda_{2}}$, while it has two zero eigenvalues and a pair on the imaginary axis for $k>1-\frac{I_{3}}{\lambda_{2}}$, as we expect from the Energy-Casimir analysis. For $r>0$ we apply the Routh test. Writing (2.7) as

$$
\lambda^{3}+\rho_{1} \lambda^{2}+\rho_{2} \lambda+\rho_{3}
$$

the Routh criterion for having all eigenvalues of the system in the left half-plane (see e.g. Gantmacher [7]) is that there should be no changes of sign in the sequence

$$
\begin{aligned}
& \left\{1, \rho, \frac{\rho_{1} \rho_{2}-\rho_{3}}{\rho_{1}}, \rho_{3}\right\} \\
= & \left\{1, r \nu, \frac{M^{2} a_{3}}{I_{3}}\left[k-\frac{1}{I_{3} \nu}\right],\right. \\
& \left.M^{2} a_{3} r\left[\frac{1}{I_{3}^{2}}-\mu \nu\right]\right\} .
\end{aligned}
$$

This requires $k-\frac{1}{I_{3} \nu}>0$ and $\frac{1}{I_{3}^{2}}-\mu \nu>0$, yielding

$$
\begin{gathered}
k>\frac{J_{3}}{J_{3}+I_{3}} \\
\left(I_{3}-I_{2}\right)+\left(J_{3}-J_{2}\right)>0 .
\end{gathered}
$$

Thus we have
Theorem 2.2. The system (2.6) is asymptotically stable under the conditions (2.11), (2.12).

Combining (2.11) and (2.12) yields precisely the condition $k>1-\frac{I_{3}}{\lambda_{2}}$ of Theorem 2.1.

This example is suggestive of the general technique for assessing asymptotic stability via the EnergyCasimir method. We will discuss this in detail in a forthcoming publication.

\section{The Energy-Momentum Method}

In order to analyze stability we turn now to the Energy-Momentum method where, as mentioned earlier, the analysis takes place in the spatial frame of reference.

In the Energy-Momentum method (Marsden et. al. [11]) one considers a symplectic manifold $(P, \Omega)$ and a Lie group $G$ acting symplectically on $P$ with equivariant momentum mapping $\mathbb{J}: P \rightarrow \mathfrak{g}^{*}$. If $H:$ $P \rightarrow \mathbb{R}$ is a $G$-invariant Hamiltonian, $z_{e} \in P$ is called a relative equilibrium if there is a $\xi \in \mathfrak{g}$ such that for all $t \in \mathbb{R}, z(t)=\exp (t \xi) z_{e}$, where $z(t)$ is the dynamical orbit of $X_{H}$, the Hamiltonian vector field of $H$ with $z(0)=z_{e}$. Now one can show that $z_{e}$ is a relative equilibrium if and only if there is a $\xi \in \mathfrak{g}$ such that $z_{e}$ is a critical point of $H_{\xi}(z)=H(z)-$ $\left\langle J(z)-\mu_{e}, \xi\right\rangle$ where $\mu_{e}=J\left(z_{e}\right)$.

The key to the Energy-Momentum method is that one can find a subspace $S \subset \operatorname{ker} d J\left(z_{e}\right)$ such that definiteness of $\delta^{2} H_{\xi}\left(z_{e}\right)$ restricted to $S$ yields stability, and, moreover, this second variation blockdiagonalizes on $S$. For this analysis one considers systems where $P=T^{*} Q$, the cotangent bundle of $Q$, the configuration space of a given mechanical system with Hamiltonian $H=K(q, p)+V(q)$, where $K$ is a quadratic form in the momentum variables $p$, and $V(q)$ is the potential energy.

In this paper we will not describe the details of the block diagonalization, (we refer to Marsden et. al. [11]), but state merely that one can reduce $\delta^{2} H_{\xi}$ to a block diagonal matrix of the form

$\left[\begin{array}{c|c|c}A & 0 & 0 \\ \hline 0 & \Lambda & 0 \\ \hline 0 & 0 & M^{-1}\end{array}\right]$

where $A$ is a positive definite co-adjoint orbit block $(2 \times 2$ in the case of $G=S O(3)), \Lambda$ corresponds to the 
second variation of the augmented potential energy and $M^{-1}$ to the inertia matrix.

Now to get the linearized dynamics we need the corresponding symplectic form for the linearized dynamics, which is given by

$$
\Omega=\left[\begin{array}{c|c|c}
L & C & 0 \\
\hline-C^{T} & S & 1 \\
\hline 0 & -1 & 0
\end{array}\right]
$$

where $S$ is skew-symmetric. We remark that in (3.1) and (3.2) the upper block corresponds to the "rotational" dynamics ( $L$ is in fact the co-adjoint orbit symplectic form for $G$ ) while the two lower blocks correspond to the "internal" dynamics. In (3.2) $C$ represents coupling between the internal and rotational dynamics, while $S$ gives the Coriolis or gyroscopic forces.

The corresponding linearized Hamiltonian vector field is then given by $X_{H}=\left(\Omega^{-1}\right)^{T} \nabla H=$ $\left(\Omega^{-1}\right)^{T} \delta^{2} H_{\xi}$, which a computation (that we omit here) reveals to be

$X_{H}=\left[\begin{array}{c|c|c}-L^{-1} A & 0 & -L^{-1} C M^{-1} \\ \hline 0 & 0 & M^{-1} \\ \hline-C^{T} L^{-1} A & -\Lambda & -\widetilde{S} M^{-1}\end{array}\right]$

where $\widetilde{S}=S+C^{T} L^{-1} C$.

To add damping to the "internal" variables (but not the rotational variables) we add a term $-R M^{-1}$ to the $(3,3)$ block. This $R$ is the Rayleigh dissipation matrix.

We restrict ourselves here to consideration of the case $G=S^{1}$, an abelian group, in which case the $(1,1)$ block $A$ vanishes. This corresponds, for example, to the analysis of planar rotating systems, such as in $\mathrm{Oh}$ et. al. [13]. The general case will be discussed in a forthcoming paper.

Taking $M=I$, we obtain the linear system

$$
\begin{aligned}
& \dot{x}=v \\
& \dot{v}=-\Lambda x-R v+S v
\end{aligned}
$$

where $R=R^{T} \geq 0$ is the Rayleigh dissipation matrix and $S=-S^{T}$ gives the gyroscopic forces in the system. Note that for

$$
\begin{aligned}
H & =\frac{1}{2} v^{2}+\frac{1}{2} x^{T} \Lambda x \\
\frac{d H}{d t} & =-2 \mathcal{R}, \quad \mathcal{R}=\frac{1}{2} v^{T} R v
\end{aligned}
$$

where $\mathcal{R}$ is the Rayleigh dissipation function.

Systems of this type were analyzed by Chetaev and Thompson (Lord Kelvin) and we shall call this the Chetaev-Thompson normal form.

Two questions of interest to us that were analyzed by Chetaev are: a) if the system (3.4) is stable for $R=S=0$, does it retain stability for $S \neq 0, R>0$; and $b$ ) if the system is only gyroscopically stable, i.e. it is unstable for $R=S=0$ and neutrally stable for $S \neq 0$, does it become unstable for $R>0$ ?

The answer to both these questions is in the affirmative, and we shall concern ourselves here with the latter question.

This question is of interst to us because it indeed shows that by examining the $\Lambda$-block of $\delta^{2} H_{\xi}$ one can deduce instability for the linearized system without finding the spectrum of the system.

It is instructive to examine first the two degreesof-frreedom system

$$
\begin{array}{r}
\ddot{x}-g \dot{y}+\gamma \dot{x}+\alpha x=0 \\
\ddot{y}+g \dot{x}+\delta \dot{y}+\beta y=0
\end{array}
$$

with $\gamma \geq 0, \delta \geq 0$. Here $g$ represents the intensity of gyroscopic forces, $\gamma$ and $\delta$ the damping, and $\alpha$ and $\beta$ the stiffness. (See also Baillicul and Levi [3].)

The characteristic polynomial for the system is

$$
\begin{aligned}
p(\lambda) & =\lambda^{4}+\lambda^{3}(\gamma+\delta)+\lambda^{2}\left(g^{2}+\alpha+\beta+\gamma \delta\right) \\
& +\lambda(\gamma \beta+\delta \alpha)+\alpha \beta
\end{aligned}
$$

For $\gamma=\delta=0$, it is simple to calculate the eigenvalues and one deduces that

(i) for $\alpha, \beta>0$ the system is spectrally stable

(ii) for $\alpha>0, \beta<0$ the system is unstable (a special case of Oh's lemma - see later)

(iii) for $\alpha<0, \beta<0$ the system is spectrally stable for $g^{2}+(\alpha+\beta) \geq 2|\sqrt{\alpha \beta}|$, unstable otherwise.

To analyze the dissipative case we employ Routh's scheme as in section 2. We can show in fact

Proposition 3.1. For $\alpha, \beta<0$ and one of $\gamma, \delta>0$ the null solution is unstable for system (3.7).

Proof. Write the characteristic polynomial as

$$
\lambda^{4}+\rho_{1} \lambda^{3}+\rho_{2} \lambda^{2}+\rho_{3} \lambda+\rho_{4}
$$


as before.

The number of right half plane eigenvalues then equals the number of sign changes in the sequence

$$
\begin{aligned}
& \left\{1, \rho_{1}, \frac{\rho_{1} \rho_{2}-\rho_{3}}{\rho_{1}},\right. \\
& \left.\quad \frac{\rho_{3} \rho_{1} \rho_{2}-\rho_{3}^{2}-\rho_{4} \rho_{1}^{2}}{\rho_{1} \rho_{2}-\rho_{3}}, \rho_{4}\right\} .
\end{aligned}
$$

From the assumptions of the theorem $\rho_{1}=(\gamma+$ $\delta)>0, \rho_{2}=\left(g^{2}+\alpha+\beta+\gamma \delta\right)>0 \rho_{3}=(\gamma \beta+\alpha \delta)<0$ and $\rho_{4}=\alpha \beta>0$. This yields the sign sequence $\{+,+,+,-,+\}$, giving the result.

Consider now the general case. We have the following result, which is due to Chetaev. Our proof is a slight modification of his which extends to infinite dimensions (see below).

Proposition 3.2. Suppose $\Lambda$ has one or more eigenvalues in the left-half-plane. Then the system (3.4) is unstable.

Proof. We use a Lyapunov instability argument. Let

$$
\begin{aligned}
W & =H+\beta B x \cdot v \\
& =\frac{1}{2}\left(v^{T} x^{T}\right)\left[\begin{array}{c|c}
1 & \frac{\beta}{2} B \\
\hline \frac{\beta}{2} B^{\prime} & \Lambda
\end{array}\right]\left(\begin{array}{l}
v \\
x
\end{array}\right),
\end{aligned}
$$

$B$ and $\beta$ to be determined. Then

$$
\begin{aligned}
& \dot{W}=\frac{1}{2}\left(v^{T} x^{T}\right) \\
& {\left[\begin{array}{c|c}
R-\beta B & \frac{\beta}{2}(R+S) B \\
\hline \frac{\beta}{2}(R+S) B & \beta \Lambda B
\end{array}\right]\left(\begin{array}{l}
v \\
x
\end{array}\right) .}
\end{aligned}
$$

Now choose $B=\Lambda^{-1}$. Then, for $\beta$ sufficiently small, $\dot{W}$ is negative definite, but $W$ has at least one negative eigendirection. Hence by Lyapunov's instability theorem we have nonlinear instability.

Remark. The proof goes through for (3.4) defined on a separable Hibert space for $\Lambda^{-1}$ compact and $(R+S) \Lambda^{-1}$ bounded.

We note however that the above result proves nonlinear instability of the linear system (3.4), not spectral instability. It is easy to construct an example of such a system without dissipation (i.e. when $R=0$ ) which is nonlinearly unstable but has eigenvalues on the imaginary axis. In such a case we have no information on the stability of the nonlinear system which has (3.4) as its linearization. Spectral instability will be discussed in a forthcoming paper.

However, if $\Lambda$ has odd index we can deduce spectral instability by the following argument (see Chetaev [6] and also Oh [12]).

The characteristic polynomial of the system (3.4). is

$$
\Delta(\lambda)=\operatorname{det}\left(\lambda^{2} I+\lambda(S-R)+\lambda\right) .
$$

For $\lambda=0$ we have $\Delta(0)=\operatorname{det}(\Lambda)<0$. Now as $\lambda \rightarrow$ $\infty, \Delta(\lambda) \rightarrow \lambda^{2} I \rightarrow+\infty$. Hence there exists a positive (real) $\lambda^{*}$ such that $\Delta\left(\lambda^{*}\right)=0$, i.e. there exists a right half plane eigenvalue and we have spectral instability.

\section{REFERENCES}

[1] R. Abraham and J. E. Marsden, Foundations of Mechanics, Addison-Wesley, 1978.

[2] V. Arnold, Sur la geometrie differentielle des groups de Lie de dimension infinie et ses applications à l'hydrodynamique des fluids parfait, Ann. Inst. Fourier, Grenoble 16, 314-361, 1966.

[3] J. Baillieul and M. Levi, Constrained Relative Motion in Rotational Mechanics, to appear in Arch. Rat. Mech. Anal., 1991.

[4] A. M. Bloch and J. E. Marsden, Stabilization of rigid body dynamics by the energy-Casimir method, Systems and Control Letters 14, 341346, 1990.

[5] A. M. Bloch, P. S. Krishnaprasad, J. E. Marsden and G. Sanchez de Alvarez, Stabilization of rigid body dynamics by internal and external torques, submitted for publication, also Systems Research Center Technical Report TR-9058,1990

[6] N. G. Chetaev, The Stability of Motion, Pergamon Press, 1961.

[7] R. R. Gantmacher, The Theory of Matrices, Vols. I and II, Chelsea, 1959.

[8] D. D Holm, J. E. Marsden, T. Ratiu and A. Weinstein, Nonlinear stability of fluid and plasma equilibria, Physics Reports 123, 1-116, 1985.

[9] P. S. Krishnaprasad, Lie-Poisson structures, dual-spin spacecraft and asymptotic stability, Nonlinear Analysis, TMA 9, 1011-1035, 1985.

[10] P. S. Krishnaprasad and J. E. Marsden, Hamiltonian structures, and stability for rigid bodies with flexible attachments, Arch. for Rat. Mechanics and Analysis, 98, 73-93, 1987.

[11] J. E. Marsden, J. C. Simo, D. Lewis and T. A. Posbergh, Block diagonalization and the 
energy-momentum method, Contempory Math. A.M.S. 97, 297-314, 1990.

[12] Y-G. Oh, A stability criterion for Hamiltonian systems with symmetry, Journal of Geometry and Physics 4, 163-182, 1987.

[13] Y. G. Oh, N. Sreenath, P. S. Krishnaprasad and J. E. Marsden, The Dynamics of Coupled Planar Rigid Bodies, Part 2: Bifurcations, Periodic Solutions and Chaos, Dynamics and Differential Equations, 1-32, 1989.

[14] J. C. Simo, T. A. Posbergh and J. E. Marsden, Nonlinear stability of elasticity and geometrically exact rods by the energy-momentum method Physics Reports, to appear.

[15] A. J. van der Schaft, Stabilization of Hamiltonian Systems, Nonlinear Analysis, TMA, 10, 1021-1035, 1986. 